\title{
Adaptive Adjustment of Feature Weight Coefficients Based on Genetic Algorithm in Image Retrieval
}

\author{
Zhihui Wang1,a, Xiaoli Ge ${ }^{1,2, b}$, Jinlin Li1,c, Qila Sa1,d, Wenbo Xu1,e, Yuejiao Fan ${ }^{1, f}$, \\ Yiqun Zhao ${ }^{1, g}$ \\ ${ }^{1}$ College of Electronic Information and Engineering, Inner Mongolia University, Hohhot 010021, \\ China \\ ${ }^{2}$ Branch Ordos of China Telecom Corporation Ltd, Ordos, 017000, China \\ a email: wzhbit2007@163.com, bemail: gexiaoli1204@163.com, 'cemail: 15127519681@163.com

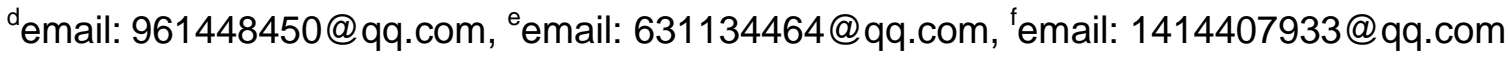 \\ gemail:597830980@qq.com
}

Keywords: CBIR (content-based image retrieval); GA(Genetic Algorithm); Adaptive Weights

\begin{abstract}
It is worth studying that how to utilize image features so as to achieve the satisfied result of CBIR (content-based image retrieval). To solve this problem, this paper proposes an adaptive image retrieval algorithm based on color feature and texture feature, in which the two kinds of features are combined and the weight coefficients of them are determined with genetic algorithm. Genetic algorithm, starting from solving practical problems, constructs an initial population with the potential solutions of practical problems. In the proposed algorithm, firstly, the initial population consisted with the weight coefficients of texture features (or color ones) is randomly generated; then, selection, crossover, and mutation are operated so that each new generation of population is gradually closed to the optimal solution; finally, the adaptively adjusted weight coefficients are obtained. Experimental results show that when fusing color feature and texture feature in image retrieval, the introduction of genetic algorithm, by which determine the weight coefficients of two kinds of features, makes both the recall ration and precision ratio of retrieval are improved. The proposed algorithm of image retrieval combined with genetic algorithm can automatically set the optimal weights of the image features according to the different image to be retrieved submitted by users, and can basically achieve the ideal suitable weights and output the relative ideal retrieval results.
\end{abstract}

\section{Introduction}

With changes in application requirements, how to retrieve the special image from the massive image database, is becoming more and more important. Traditional TBIR (text based image retrieval) technology has many deficiencies, and CBIR (content-based image retrieval) using visual characteristics of the image itself can accurately find the desired image from a number of image library with high-efficient. So CBIR has become the hotspot [1].

CBIR mainly utilizes the distance measure function, which is calculated by the visual features or semantic features extracted from the detected image and the images in the database, to match the similarity. Finally, the retrieval results will be given to the user in accordance with the order of the similarity.

Image features include color feature, texture feature, shape feature and so on, and color feature is one of the essential visual information. In all the underlying visual features of image, color is the most significant and stable visual one compared to the other features, such as texture, shape contour and so on. Scholars at home and abroad have carried out extensive research on the retrieval algorithm based on the underlying feature of the image. Reference [2] defines a color similarity metric, and adopted it in CBIR; [3] proposed a concept of weighted color histogram based on color contrast of multi-scale neighborhood. 
Texture feature is another important visual feature of image. An algorithm of image retrieval based on texture feature is proposed in [4].

In recent years, many researchers have noticed that CBIR cannot simply uses the color, texture, such as a feature of CBIR often unable to obtain good retrieval effectiveness and to the comprehensive utilization of the color feature and texture characteristics of image features for CBIR of extensive research and discussion [5-8]

However, how to reasonably comprehensive utilization of multi feature of image is a crucial problem. In this paper, a weighted fusion using color and texture features of image retrieval algorithm, in the algorithm into the genetic algorithm to adaptively determine their optimal weight coefficient.

\section{Key Technologies of Genetic Algorithm}

Genetic algorithm (GA) that is a simulation of the process of biological evolution is a kind of method that based on the principle of survival of the fittest to solve practical problems.

In genetic algorithm, a potential solution set is constructed into an initial population, in which each individual is gene encoded. After the initial population is determined, it is necessary to simulate the process of biological evolution. In the evolution process, a new generation of population gradually closed to the optimal solution is created through the population selection, crossover and mutation operation, produced, finally the practical problem can be solved.

The key technology of genetic algorithm [9] can be summed up as a seven-tuple:

$\boldsymbol{G A}=(\boldsymbol{C}, \boldsymbol{f}, \boldsymbol{s}, \varphi, \tau, \psi, \boldsymbol{T})$

Among them, $C$ denotes individual encoding mode, $f$ on behalf of fitness function, $S$ represents the initial population, $\varphi$ denotes selection operator, $\tau$ represents crossover operator, $\psi$ represents mutation operator, and $T$ denotes the termination condition of algorithm. Next, we will introduce the several key technologies one by one.

\section{A. Coding Mode $C$}

Coding mode is the first step and the base in the genetic algorithm. Only biological genetic coding determined, biological traits can be identified. Binary coding, is one of commonly used coding methods. Binary code is relatively simple, it adopts a binary 0,1 string to represent an individual, 1 denotes having certain characteristics, and 0 does not having.

\section{B. Fitness Function $f$}

In the image retrieval process based on GA, the main basis for judgment is the fitness value. Therefore, choose appropriate fitness function is very important. It not only affects the convergence speed of genetic algorithm, but also influences the ability to find the global optimal solution. In the proposed algorithm, the fitness function of each individual is set:

$$
f_{i}=\sum_{i=1}^{n u m} \sqrt{\frac{R^{2}+P^{2}}{2}}
$$

where $R=a / k, P=a / z ; a=\sum_{i=1}^{z} v_{i}$,

$v_{i}=\left\{\begin{array}{ll}1, & \text { when rertievaled image is the relevated image } \\ 0, & \text { otherwise }\end{array} ;\right.$

num is the number of images in library, $R$ on behalf of recall; $P$ represents precision of; $a$ denotes the number of correlated images retrieved; $k$ represents the number of correlated images in the library. Since there are 10 images for each kind of image in our algorithm, $k=10 ; \quad z$ is the number of most relevant images (In the proposed algorithm, the first 9 of the most relevant images retrieved are outputted as a result. So $z$ equals 9.). And $v$ denotes the judgment result, by which 1 means that the retrieved image is relevant, 0 is not. 


\section{Initial Population $S$}

Initial population refers to a given population at the beginning of biological evolution process. Its size directly affects the retrieval effectiveness. If the initial population is larger, the opportunity of obtaining the optimal solution of is higher (through genetic manipulation). However, at the same time, the times of evaluating fitness will be increased, the amount of calculation is increased. Due to the survival probability of a body is proportional to fitness degree, so a lot of unnecessary of low probability calculation will be produced, which would seriously affect the computational efficiency.

\section{Selection Operator $\varphi$}

Selection, also known as replication or regeneration, is to simulate the process of natural selection. It selects outstanding individual in a population used to produce the next generation. The selection operation is based on the individual fitness value. The greater the degree of adaptation is, the higher probability being selected is. In this paper, fitness proportional method is used to select the excellent offspring.

Fitness proportional method, also known as roulette method, is currently the most commonly used selection method. Its basic principle which is shown in fig. 1 is individual fitness degree is proportional to the chosen probability.

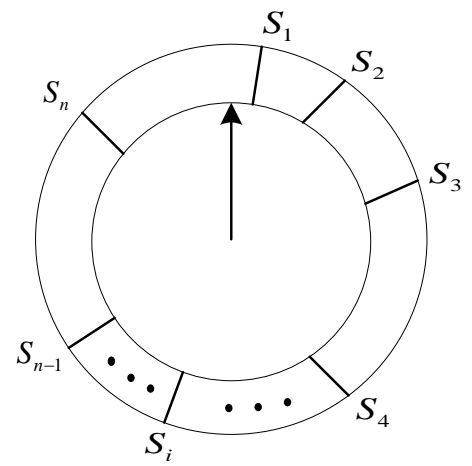

Fig.1. Roulette wheel selection

In fig.1, the scale on the circular ring represents the cumulative probability of each individual. The process of selecting is that the center pointer does not move, the outer ring rotates freely to stop, and the designated position of the pointer is the selected individual.

\section{E. Crossover Operator $\tau$}

Crossover, is to exchange the part of genes of the two father individuals, and then through the gene recombination to generate new individuals. If there are two chromosomes S1 and S2, the binary encoding of the single point crossover and two points crossover are as follows(In the single point crossover, we select the 4th bit as the crossover point; In the two points crossover operation, we take 2nd and 5th as the crossover point):

$$
\begin{aligned}
& S_{1}=0100 \vdots 101 \rightarrow S_{1}^{\prime}=0100 \vdots 100 \\
& S_{2}=1100 \vdots 100 \rightarrow S_{2}{ }_{2}=1100 \vdots 101 \\
& S_{1}=01 \vdots 001 \vdots 01 \rightarrow S_{1}^{\prime}=11 \vdots 001 \vdots 00 \\
& S_{2}=11 \vdots 001 \vdots 00 \rightarrow S_{2}{ }_{2}=01 \vdots 001 \vdots 01
\end{aligned}
$$

F. Mutation Operator $\psi$

Mutation is also an important step in the genetic process. In the genetic operation, a gene is randomly selected as a genetic change one, through gene turnover to generate new individuals. For example, the mutation of the 4th bit encoding when the binary code is as follows:

$S_{1}=010 \hat{\mathbf{0}} 101 \rightarrow S_{1}^{\prime}=010 \hat{\mathbf{1}} 101$

\section{G. Termination Condition of Algorithm $T$}

Generally, the termination condition of the algorithm $T$ can be divided into the following [10]: 
a. To achieve the maximum number of iterations. According to the complexity of the problem, it is often set between 100-500. This paper sets the number of iterations for the 300 .

b. The solution has reached an acceptable range.

c. The optimal solution has no change or only small change.

d. Has reached the maximum running time.

\section{Implementation Strategy of Adaptive Feature Weights Based on GA in Image Retrieval}

This paper presents a multi-features image retrieval algorithm, which is based on the color feature and texture feature. Moreover, GA is adopted to determine the weight distribution of the two kinds of features in the retrieval process.

A. Overall Description of the Proposed Algorithm

Color moments proposed by Stricker [11] describe color statistical distribution characteristics of a kind of effective color features. It has be proven that dividing the image into blocks before calculating color moment can improve the precision and recall of retrieval system [12].

In this paper, color feature similarity measurement is realized based on block color moment.

When it comes to texture features, GLCM (gray level co-occurrence matrix) is a commonly used method that the texture of image is described through studying the related characteristics in gray space [13]. But for an image with relatively large number of gray level, the amount of computation needed for constructing GLCM will be more. In the proposed algorithm in this paper, Canny operator is used to detect the edge of original image. Then, GLCM corresponding to the edge image is constructed. In this way, it can not only preserve the texture characteristics of the original image, but also greatly reduce the complexity and computational time of constructing GLCM.

The overall structure of the proposed algorithm is shown in fig.2.

\section{B. Strategy of Adaptive Adjustment of Feature Weights}

In this retrieval algorithm, assuming that $D_{c}$ and $D_{t}$ are color-feature and texture-feature similarity distance between query image $Q$ and any image $I$ in retrieval library respectively, $w_{C}$ and $w_{t}$ are corresponding weight coefficients. Then, we defined a weighted sum of $D_{c}$ and $D_{t}$ :

$$
D=\frac{w_{C}}{w_{C}+w_{t}} \cdot D_{C}+\frac{w_{t}}{w_{C}+w_{t}} \cdot D_{t}
$$

How to decide $w_{c}$ and $w_{t}$ in (6)? We adopt GA here. The strategy of adaptive adjustment of feature weights is as follows:

Step1: Randomly generate initial population $s_{0}=\left(C_{1}, C_{2}, C_{3} \cdots C_{N}\right), C_{i}=w_{t}, w_{c}=1-w_{t}$. Each individual $C_{i}$ is composed of a 7-bit binary string which is randomly generated.

Step2: Calculate fitness function of individual by equation (2). Judge whether it meets the algorithm terminates condition T. If meet, output the best individual and the optimal solution that is the optimal feature weight, and terminate the computation; If not, turn to Step3.

Step3: Select the regeneration of the individual. Re-product the excellent individuals using Fitness proportional method, eliminate the individual that is not suitable, and save the best individual, so as to select the weights that can output the optimal feature weight finally.

Step4: Generate new individuals using the single point crossover method and according to a certain probability of crossover $P_{c}$. The crossover probability is generally selected in the range of $0.4 \sim 0.99$.

Step5: Generate new individuals according to a certain probability of mutation of $P_{m}$. The probability of mutation is generally selected in the range of $0.001 \sim 0.1$.

Step6: Generate a new generation of population, and obtain a new set of characteristic weight values. Return to Step2 until the termination condition $T$ is satisfied. 


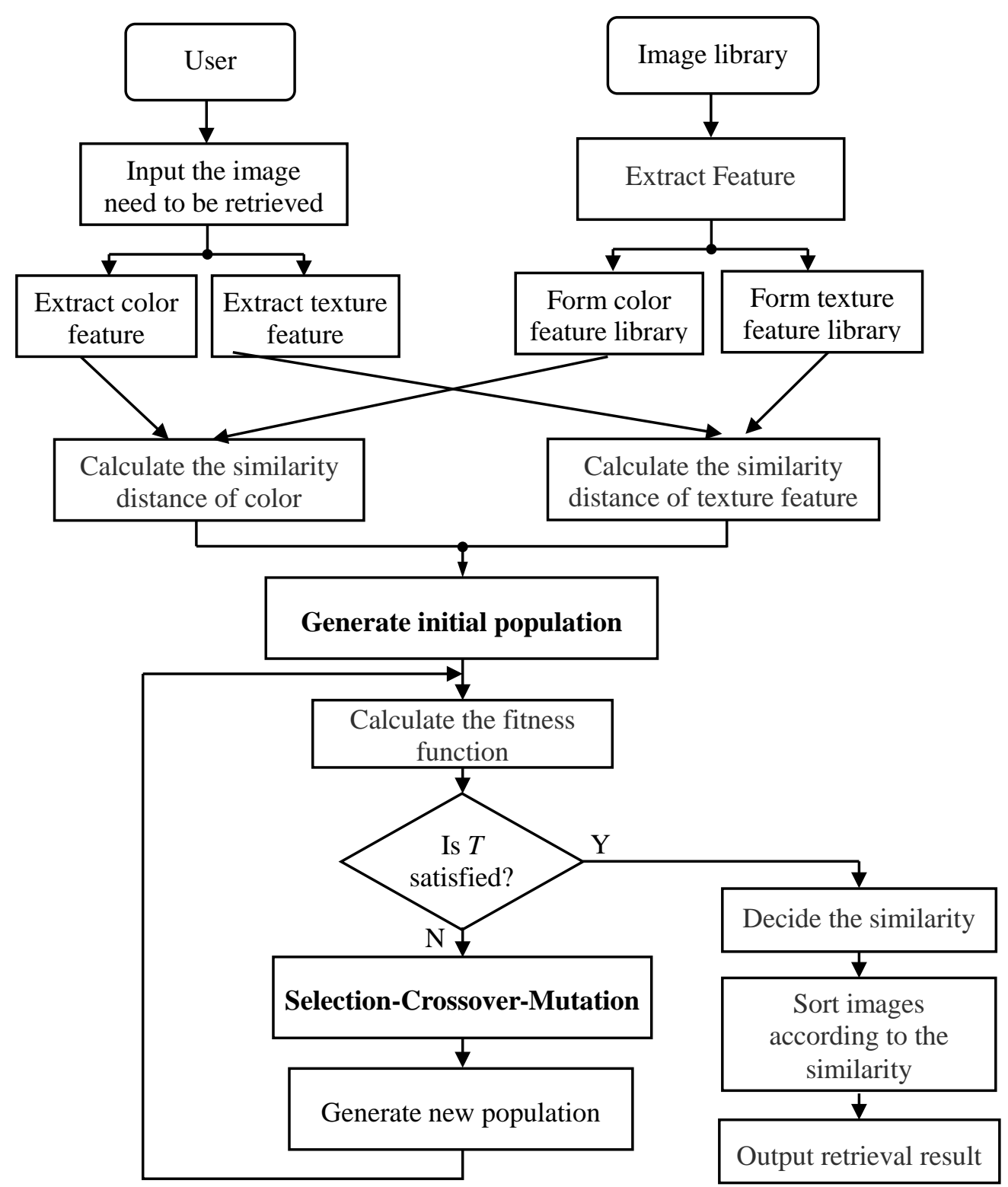

Fig.2. Flow chart of adaptive image retrieval algorithm based on GA

\section{Experimental results}

In order to verify the performance of the proposed algorithm, we use MATLAB as a development tool for image processing and simulation. In the experimentation, we select 40 images in test library coral, including four kinds of images: bus, flowers, buildings, and Africans. There are 10 image in each kind. It should be noted that these 10 images are referred to as the relevant images. In the design and implementation of retrieval system, the first 9 images which are most similar will be displayed as retrieval results. 


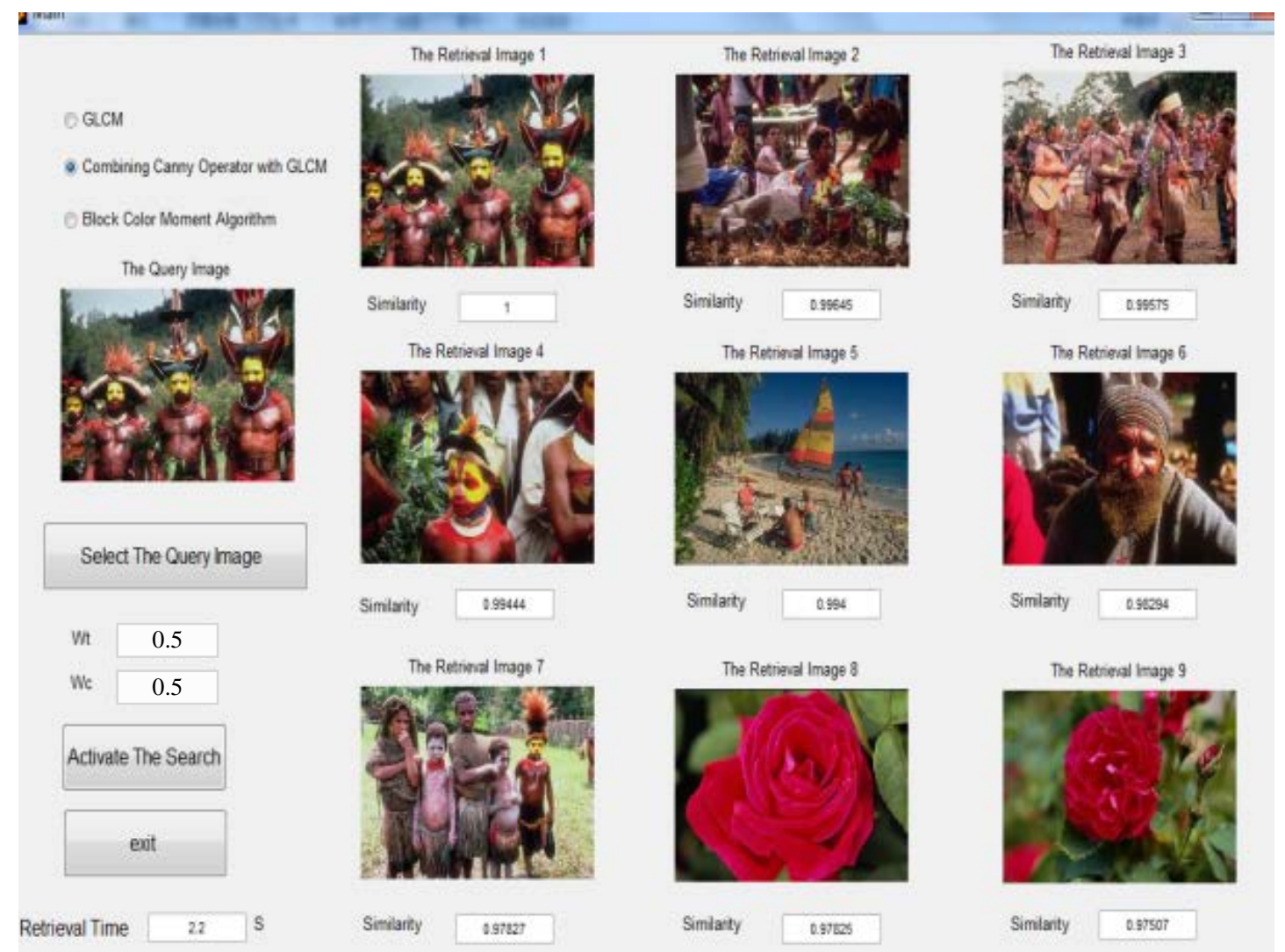

(a) Retrieval by fixed feature weight coefficients

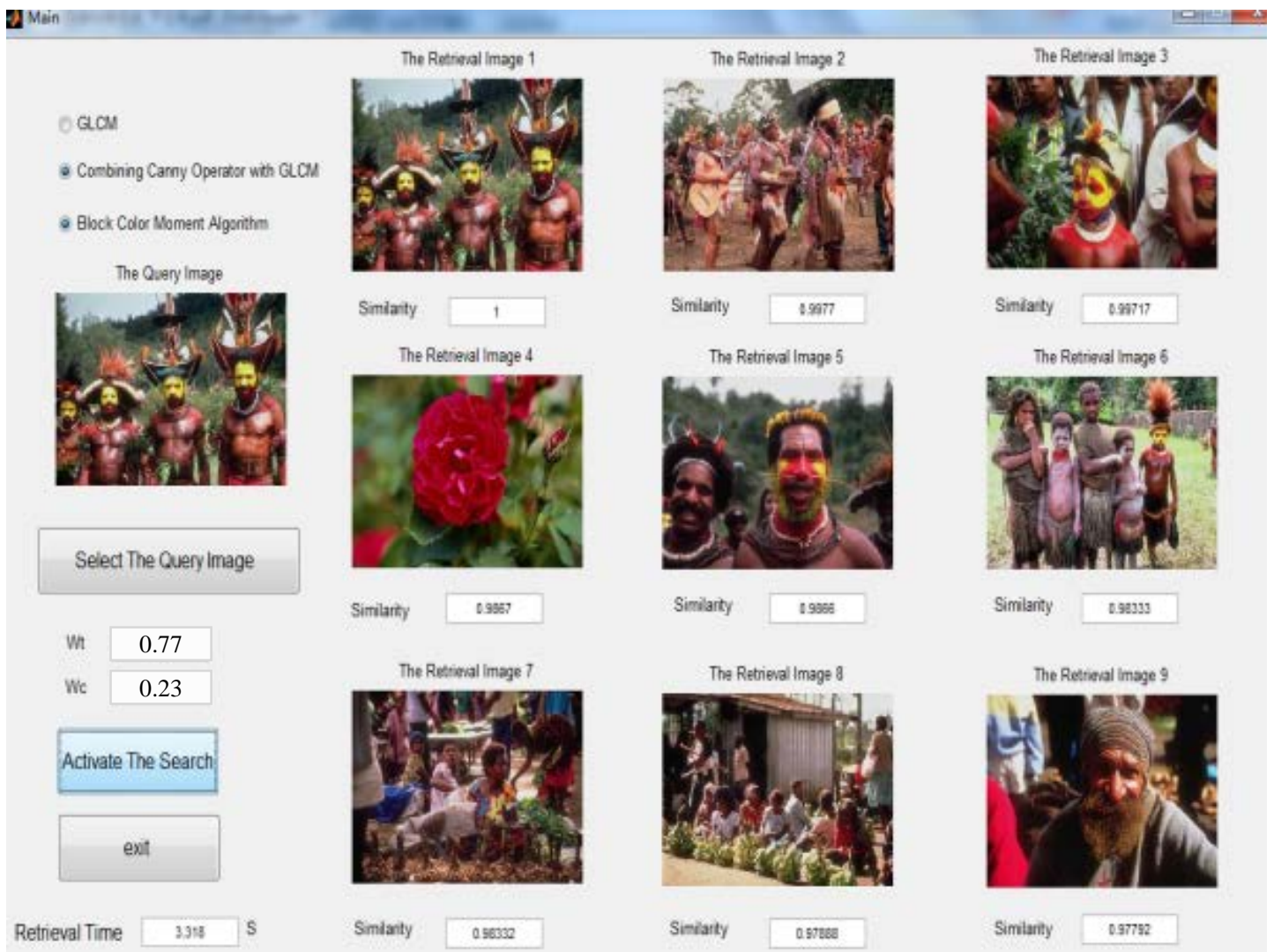

(b) Retrieval by adaptive feature weight coefficients based on GA

Fig.3. The experimental results

\section{Conclusion}

This paper put forward a method using GA (genetic algorithm) to deal with how to adaptively 
determine the weight coefficients in the image retrieval based on multiple features. In this method, a randomly generated initial population is texture feature weight $w_{t}$; a new population is generated after selection, crossover, and mutation operation; optimal weight coefficients of the color feature and texture feature are obtained until the termination condition $T$ is meet. Experimental results show that the algorithm is reasonable and feasible. Compared with the method using fixed weight coefficients in multi-features image retrieval, recall ratio and precision ratio are all improved. Certainly, there are something still worth studying and exploring.

\section{Acknowledgement}

In this paper, the research was sponsored by the National Natural Science Foundation of China (Grant No.61261003), and the Natural Science Foundation of Inner Mongolia Autonomous Region of China (Grant No.2015MS0610).

\section{References}

[1] X Y Wu, Y He, L Yang, et al. Binary image retrieval based on improved shape context algorithm [J]. Opt. Precision Eng., 2015: 23(1): 302-309.

[2] X D Gu, C Yang. Application of new color similarity measurement method in image retrieval [J]. Chinese Journal of Scientific Instrument, 2014:35(10): 2286-2292.

[3] Q Zhao, J Cao, Y Hu. Image retrieval based on color-spatial distributing feature [J]. Multimedia and Signal Processing Communications in Computer and Information Science, 2012:34(6): 79-86.

[4] KEKER H B, THEPADE S D. Image retrieval using color-texture features extracted from walshlet pyramid [J]. ICGST International Journal on Graphics, Vision and Image Processing, 2010 (10) 9-18.

[5] Y Zhou, F Z Zeng, H M Zhao, et al. An image retrieval method based on meticulous sparsity adaptive matching pursuit algorithm [J]. Acta Electronica Sinica, 2014 (12) 2457-2466.

[6] W Shi, X F Zhu. Image retrieval based on Contour reconstruction and feature point chord length [J]. Journal of Software, 2014:25(7): 1557-1569.

[7] H D Zhu, W Song. Image retrieval based on difference between pixels \& motif matrix [J]. Application Research of Computers, 2015:32(10): 3151-3155.

[8] F Xue, J Gu, G Y Cui, et al. ROI selection and image retrieval method based on contribution matrix of SURF features[J]. Journal of Computer-Aided Design \& Computer Graphics, 2015 (7)1271-1277.

[9] F Yao, L N Xing, J F Li, et al. Knowledge-based genetic algorithm to the double layer capacitated arc routing problems[J]. Systems Engineering-Theory \& Practice, 2014:34(1): 239-247.

[10] S R Qu, H H Yang. Infrared image segmentation based on PCNN with genetic algorithm parameter optimization[J]. High Power Laser and Particle Beams, 2015: 27(5): 32-37.

[11] Stricker M, Orengo M. Similarity of Color Image [C]. In Proc. Of SPIE Storage and Retrieval for Image and Video Database, 1995, 2420: 381-392.

[12] Y W Yu, X H Zhou, H J Xu. Scale-invariant moment based robust image copy detection in DCT domain [J]. Journal of University of Electronic Science and Technology of China, 2013(4) 603-608.

[13] H Wang F, Y Wang T, H Chai. State-of-the-Art on texture-based well logging image classification [J]. Journal of Computer Research and Development, 2013: 50(6): 1335-1348. 